\title{
B CHROMOSOMES SLOW DEVELOPMENT IN A GRASSHOPPER
}

\author{
A. W. HARVEY* and G. M. HEWITT \\ School of Biological Sciences, University of East Anglia, Norwich, U.K.
}

Received 1.ii. 79

\section{Summary}

\begin{abstract}
Some populations of the grasshopper, Myrmeleotettix maculatus, are polymorphic for the presence of $\mathrm{B}$ chromosomes. In a culture derived from a population near Aberystwyth in Wales, it was found that grasshoppers with two or more B chromosomes took longer to develop from egg diapause to adult than those with one or none. There was no significant difference between $\mathrm{OB}$ and $1 \mathrm{~B}$ grasshoppers. The significance of these findings for the maintenance of the B chromosomes is discussed.
\end{abstract}

\section{INTRODUCTION}

Two questions have preoccupied those who study B chromosomes; how is the presence of relatively large amounts of supernumerary chromatin consistent with the functioning of the genome, and do they only persist in populations because their deleterious effect on the organism is balanced by an ability to increase their frequency at cell division?

Because the term "B chromosome" embraces a heterogeneous assemblage of abnormal chromosomes, as varied in their behaviour as in their size and shape, generalisations are difficult to make. The only way forward seems to be by careful comparison between detailed case studies. One such example is Myrmeleotettix maculatus (Thunberg), a small grasshopper which lives on dry heathland and has an obligatory winter egg diapause. Populations in central and southern Britain are polymorphic for a large metacentric B chromosome (Barker, 1966; Hewitt and Brown, 1970), which is stable at mitosis (John and Hewitt, 1965). Its inheritance has been studied by singlepair crosses in the laboratory, and it has been demonstrated that there is an increase in frequency through the female and a decrease through the male (Hewitt, 1973). It has been shown that the female meiotic drive is the result of the unpaired $\mathrm{B}$ chromosome moving more often to the egg nucleus than to the polar bodies at first anaphase (Hewitt, 1976). The mechanism of $\mathrm{B}$ chromosome loss in the male is still being investigated.

The precise degree of gain and loss varies between populations, but, in all except one that have been studied, the net effect is to increase the frequency of B's in the eggs over that in the adults. As the frequencies in the adults of these populations have remained constant over several years (Hewitt, 1973; Harvey, 1977), it follows that in each population there must be selection acting on those individuals with $\mathrm{B}$ chromosomes equal and opposite to the overall gain at meiosis.

The work reported here was part of a programme to discover what effect

* Present address: Centre for Overseas Pest Research, College House, Wright's Lane, London W8 5SJ. 
B chromosomes have on $M$. maculatus in order to find out more about the selective forces that maintain the polymorphism. It compares the rate of development, from the time the egg breaks diapause until the insect fledges into an adult, in grasshoppers with no, one and two or more, $\mathrm{B}$ chromosomes.

$\mathrm{B}$ chromosomes have been found to slow growth in several plant species (see Jones, 1975, for review) and in a mealy bug, Pseudococcus obscurus (Nur,
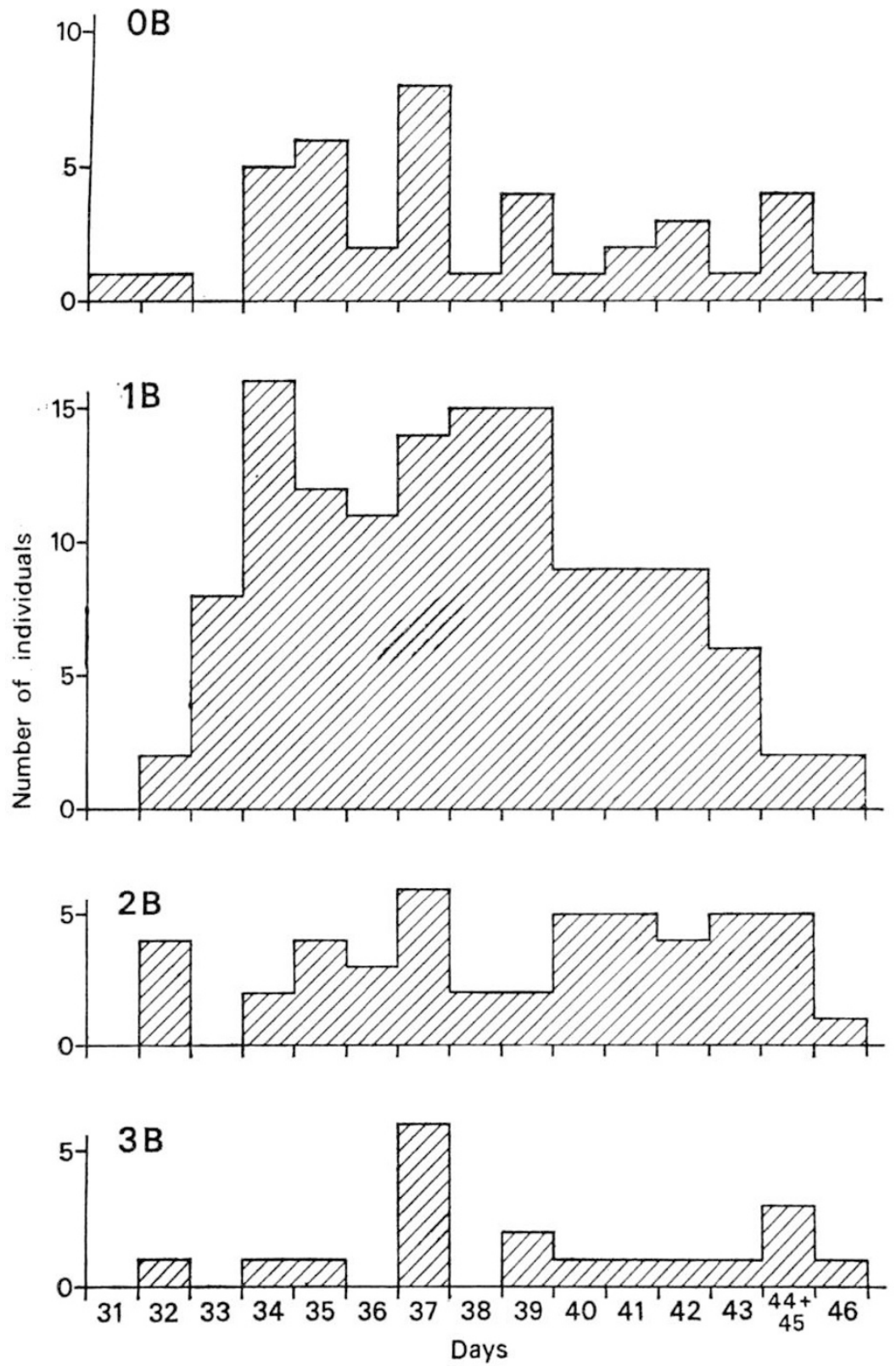

Fig. 1-The numbers of individuals of each karyotype fledging to adult each day after removal from diapause. 
1966). This is a characteristic that is very likely to affect fitness, and also, since development in insects is related to temperature, could be connected with the correlation that exists between the $\mathbf{B}$ frequency in a population and the temperature at the locality where it occurs (Hewitt and Brown, 1970; Hewitt and Ruscoe, 1971).

\section{Methods and materials}

The insects used in this experiment were from a laboratory culture derived from a population at Talybont near Aberystwyth, where the B frequency is amongst the highest known. The eggs had been kept in diapause at $4^{\circ} \mathrm{C}$ since the previous summer. The egg pods, stored in Petri dishes filled with damp sand (Kelly-Stebbings and Hewitt, 1972), were incubated at $26^{\circ} \mathrm{C}$ for 7 days. They were then transferred to a 50-litre locust cage fitted with a light bulb inside and reared to fledging.

The viscera were vivisected from the fledglings and the male testes fixed immediately in $3: 1$ ethanol : glacial acetic acid. Ovaries and gastric caeca from the females were first incubated in a hypotonic solution of colchicine in insect saline. The tissues were examined as squash preparations stained in lacto-proprionic orcein.

\section{RESUlts}

The numbers of each karyotype fledging each day are shown in fig. 1 . Table 1 shows the mean development time of each karyotype. Table 2 presents the results compressed into a $3 \times 3$ contingency table which departs from the distribution of karyotypes expected if there was no difference in rate of development $\left(\chi_{(4)}^{2}=12.9: \mathrm{P}<0.02\right)$. Applying the test to the first two columns only, one finds no significant difference between $0 \mathrm{~B}$ and $\mathbf{B}$ individuals, but if one applies it to the second and third columns a significant difference is revealed between individuals with one $\mathbf{B}$ and those with two or

TABLE 1

Mean number of days from diapause to fledging

$\begin{array}{lcccc} & \text { OB } & 1 \mathrm{~B} & 2 \mathrm{~B} & 3 \mathrm{~B} \\ \text { Male } & 37 \cdot 48 & 37 \cdot 57 & 39 \cdot 01 & 39 \cdot 20 \\ \text { Female } & 39 \cdot 08 & 37 \cdot 93 & 40 \cdot 25 & 39 \cdot 30 \\ \text { Total } & 38 \cdot 00 & 37 \cdot 75 & 39 \cdot 65 & 39 \cdot 29\end{array}$

TABLE 2

Number of grasshoppers of each karyotype fledging in successive periods

$\begin{array}{llrc}\text { Days } & \text { OB } & 1 \mathrm{~B} & 2 \mathrm{~B}+3 \mathrm{~B} \\ 31-35 & 13 & 38 & 16 \\ 36-40 & 16 & 64 & 36 \\ 41-46 & 11 & 28 & 41 \\ & 40 & 130 & 93\end{array}$

Total table: $\quad \chi_{(4)}^{2}=12.9, \mathrm{P}<0.02$

OB v. 1B: $\quad \chi_{(2)}^{2}=0.64$, not significant

1B v. $2 \mathrm{~B}+3 \mathrm{~B}: \chi_{(2)}^{2}=12 \cdot 08, \mathrm{P}<0 \cdot 01$ 
three. It should be emphasised that there are many fewer 0B's than in the other two categories, and so the ability to detect any difference is accordingly less.

\section{Discussion}

This experiment shows that, in the Talybont population, grasshoppers that have two or more B chromosomes develop more slowly from diapause to fledging than those with none or one. It does not show conclusively whether there is a difference between those without B's and those with one, but if such a difference exists, it is relatively small. It does, however, show that the effect is not simply additive, as that of two B's is disproportionately greater than that of one. Nor does it seem to conform to the "odds and evens effect " that Kirk and Jones (1970) found in rye, where odd numbers of B's had a greater effect than even numbers. They suggested that when B chromosomes are able to pair at some stage of the cell cycle they are less disruptive. But since the M. maculatus $\mathrm{B}$ is an iso-chromosome ( John and Hewitt, 1965), in genetical terms there is always an even number of supernumeraries, and this fact may have been important in its history.

Grasshoppers from Talybont were chosen for this experiment because this is a population whose $\mathrm{B}$ frequency is high enough to produce a reasonable proportion of $2 \mathrm{~B}$ individuals. However, it is likely that the effect of the $\mathrm{B}$ chromosomes varies in degree, if not in kind, between populations, as differences have been found in their level of transmission (Hewitt, 1973). It is to be expected that the rest of the genome will adapt to some extent to the presence of B chromosomes and that this may be achieved differently in different populations. In particular, it will presumably be best adapted to the karyotype that is most common in the population. At Talybont this is 1B. In populations with a lower $\mathrm{B}$ frequency, $0 \mathrm{~B}$ might be the largest class, and we might find there that the differences between $\mathrm{OB}$ and $\mathrm{IB}$ were greater. It is perhaps significant that although 3B embryos are quite commonly found in cultures from East Anglian populations, from the several hundred adults that have been examined, both from culture and from the field, only two have had three B's, while in this experiment there were 17 with 3B's in 263 adults. For this reason, we cannot simply extrapolate this result to other populations.

How might supernumerary chromosomes affect development? Fox, Hewitt and Hall (1974) showed that the B chromosome of $M$. maculatus does not incorporate $\mathrm{H}^{3}$ uridine during meiotic prophase, and so is probably not genetically active. It can be argued that a large piece of inert chromatin simply acts as a "spanner in the works". In rye, B chromosomes cause changes in the nuclear phenotype that could be interpreted as evidence of impaired function, an increase in histone content, but a decrease in total nuclear protein and RNA. But such experiments have been carried out on plants with exceptionally high numbers of B's, up to eight per cell, maintained by particular breeding techniques. One would need to be careful in extending findings of interference in cell function in those circumstances to balanced polymorphisms in populations where the genome may have adapted to some extent to the presence of supernumeraries.

It has not yet been possible to measure the effect of the B chromosome on the duration of the mitotic cycle in $M$. maculatus. This would be a very valuable piece of information, because there is evidence from plants that the 
mitotic cycle increases linearly with the DNA content of the nucleus at a rate of about 0.3 hours per picogram (Rees, 1972) and that B chromosomes lengthen the cycle at a greater rate than would be expected simply from their contribution to the DNA content. This, of course, would be expected to slow development.

It is possible that the effect of $\mathbf{B}$ chromosomes on the rate of development is of any importance in maintaining the differences between populations? There is a well established correlation between B chromosome frequency in populations and the temperature at the localities at which they occur (Hewitt and Brown, 1970; Hewitt and Ruscoe, 197I). The rate of development of grasshoppers in dependent on temperature. In Britain, grasshoppers are at the extremity of their range and their distribution is limited by temperature. It is therefore not surprising to find that a $\mathrm{B}$ chromosome which slows development is only found in the warmest part of the insect's range.

\footnotetext{
Acknowledgment.-This work was done while A. W. H. was receiving a N.E.R.C. studentship.
}

\section{REFERENCES}

BARKER, J. F. 1966. Climatological distribution of a grasshopper supernumerary chromosome. Evolution, 20, 665-667.

FOX, D. P., HEWTTT, G. M., AND HALL, D. J. 1974. DNA replication and RNA transcription of euchromatic and heterochromatic chromosome regions during grasshopper meiosis. Chromosoma, 45, 43-62.

HARVEY, A. W. 1977. The expression of supernumerary chromosomes in Myrmeleotettix maculatus (Thunberg) (Acrididae). Ph.D. thesis, University of East Anglia.

HEwITT, G. м. 1973. Variable transmission rates of a B-chromosome in Myrmeleotettix maculatus (Thunb.) (Acrididae : Orthoptera). Chromosoma (Berl.), 40, 83-106.

HEwITT, G. M. 1976. Meiotic drive for B-chromosomes in the primary oocytes of Myrmeleotettix maculatus (Orthoptera : Acrididae). Chromosoma (Berl.), 56, 381-369.

HEWITT, G. M., AND BROWN, F. M. 1970. The B-chromosome system in Myrmeleotettix maculatus. V. A steep cline in East Anglia. Heredity, 25, 363-371.

HEWITT, G. M., AND RUSGOE, G. N. E. 1971. Changes in microclimate correlated with a cline for B-chromosomes in the grasshopper Myrmeleotettix maculatus. 7. Anim. Ecol., 40, 753-765.

JOHN, B., AND HEWITT, G. M. 1965. The B-chromosome system in Myrmeleotettix maculatus (Thunb.). I. The mechanics. Chromosoma, 16, 548-578.

JONES, R. N. 1975. B-chromosome systems in flowering plants and animal species. Int. Rev. Cytol., 40, 1-100.

KIRK, D., AND JONES, R. N. 1970. Nuclear genetic activity in B-chromosome rye, in terms of the quantitative interrelationships between nuclear protein, nuclear RNA and histone. Chromosoma (Berl.), 31, 241-254.

KELLY-STEBBINGS, A., AND HEWITT, G. M. 1972. The breeding of laboratory grasshoppers. Acrida, 1, 233-275.

NUR, U. 1966. The effect of supernumerary chromosomes on the development of mealybugs. Genetics, 54, 1239-1249.

REES, H. 1972. DNA in higher plants. Brookhaven Symp. Biol., 23, 394-418. 